\title{
Linx
}

Revue des linguistes de l'université Paris X Nanterre

$62-63 \mid 2010$

Entre syntaxe et discours. Éclairages

épistémologiques et descriptions linguistiques

\section{Quelques remarques sur l'organisation syntaxique des énoncés clivés du type}

Ce n'est pas parce qu'une pratique est répandue qu'elle est sans danger

\section{Frédéric Sabio}

\section{OpenEdition \\ Journals}

Édition électronique

URL : http://journals.openedition.org/linx/1376

DOI : 10.4000/linx.1376

ISSN : 2118-9692

\section{Éditeur}

Presses universitaires de Paris Nanterre

Édition imprimée

Date de publication : 1 juillet 2010

Pagination : 89-110

ISSN : 0246-8743

Référence électronique

Frédéric Sabio, "Quelques remarques sur l'organisation syntaxique des énoncés clivés du type », Linx [En ligne], 62-63 | 2010, mis en ligne le 04 décembre 2013, consulté le 19 avril 2019. URL : http:// journals.openedition.org/linx/1376; DOI : 10.4000/linx.1376

Ce document a été généré automatiquement le 19 avril 2019

Département de Sciences du langage, Université Paris Ouest 


\section{Quelques remarques sur l'organisation syntaxique des énoncés clivés du type}

Ce n'est pas parce qu'une pratique est répandue qu'elle est sans danger

\section{Frédéric Sabio}

1 Nous proposons d'étudier dans cet article les énoncés ${ }^{1}$ tels que :

(1) c'est pas parce qu'on emploie quelques mots d'anglais que tout de suite euh notre langue euh elle va disparaitre [oral, corpaix]

(2) Et ce n'est pas parce qu'un phénomène est banalisé qu'il perd en nocivité. [écrit, CERF]

(3) Mais ce n'est pas parce qu'une pratique est répandue qu'elle est sans danger.

[écrit, CERF]

2 Ces structures en Ce n'est parce que $X$ que $Y$, qui sont manifestement apparentées aux constructions clivées, possèdent des propriétés grammaticales tout à fait spécifiques qui interdisent selon nous de les assimiler aux clivées «contrastives » les plus souvent décrites : comme nous le montrerons, les syntagmes en parce que qui apparaissent dans la première partie de ces constructions ne peuvent ni être décrits comme des subordonnées canoniques, ni être analysés comme des éléments non régis qui seraient en relation de simple association parataxique avec la construction verbale qui les suit. Nous proposerons de voir dans ces structures un type original de regroupement macrosyntaxique dont la forme est directement reliée à certains effets interactionnels de dénégation d'une inférence causale.

\section{Retour sur la notion de « dispositif clivé »}

3 Avant de dégager (au point 2 ci-dessous) certaines caractéristiques des structures illustrées dans les exemples (1-3), nous nous arrêterons sur les propriétés des énoncés clivés à effet contrastif, qui sont de loin le type le plus commenté par les linguistes ${ }^{2}$. C'est 
ce type-ci, très largement représenté dans les corpus de français oral, qui a été décrit dans le cadre élaboré par le GARS ${ }^{3}$ comme l'un des « dispositifs » de la rection verbale.

Voici en guise d'illustration l'extrait d'un dialogue oral dans lequel la construction en parce que (désormais parce que-C) qui est notée en italiques se trouve engagée dans un dispositif clivé tout à fait classique :

(4)

L2 - évidemment on voit les faiblesses de l'écrit et là on se rend compte que c'est

des gens qui écrivent très très très peu

$\mathrm{Ll}$ - $\mathrm{mh} \mathrm{mh}$ ils font beaucoup de fautes

L2 - euh c'est ça ils font une faute à chaque mot mais enfin euh c'est pas parce qu'ils

sont dans les poids lourds qu'ils font une faute à chaque mot

$\mathrm{L} 1-\mathrm{mh} \mathrm{mh}$

L2 - hein quel que soit le milieu social j'allais dire dans lequel on se tourne les gens

font des fautes [oral, corpaix. L'exemple a été légèrement simplifié]

\subsection{La clivabilité comme l'une des propriétés d'« insertion paradigmatique »}

5 La séquence parce qu'ils sont dans les poids lourds qui apparait en (4) possède toutes les propriétés d'un élément régi par un verbe recteur, en l'occurrence d'un élément qui est sous la dépendance syntaxique de faire, et peut à ce titre être considéré comme un type de complément. Nous le montrerons en nous situant dans le cadre méthodologique de l'Approche Pronominale (Blanche-Benveniste et al., 1984), qui a recours, pour attester de la présence des liens de dépendance, à un ensemble de critères d'insertion paradigmatique : on considère que la caractéristique majeure des éléments régis est qu'ils occupent une place à l'intérieur d'un paradigme ouvert par le verbe. Nous retenons les critères d'insertion paradigmatique suivants ${ }^{4}$ :

6 a- La proportionnalité à une proforme : les éléments régis par un verbe peuvent typiquement être mis en relation avec une proforme (qu'il s'agisse de pronoms au sens traditionnel du terme ou de certaines formes dotées d'un faible poids lexical), avec laquelle ils entretiennent une relation de proportionnalité : c'est bien le cas de l'énoncé ci-dessus, dans lequel la séquence parce qu'ils sont dans les poids lourds est proportionnelle à plusieurs proformes telles que les suivantes :

(5) c'est pas pour ça / pour cela / pour cette raison-ci / à cause de ça qu'ils font une

faute à chaque mot

b- La réalisation à travers plusieurs dispositifs de la rection: l'Approche Pronominale désigne par dispositif la manière dont une construction verbale agence les éléments de sa rection de manière à singulariser l'un d'entre eux en lui donnant une valeur focale particulière. On distingue ainsi les dispositifs à focalisation initiale, tels que le clivage ou le dispositif restrictif ${ }^{6}$, et les dispositifs à focalisation finale, tels que le pseudo-clivage ou le «Sidispositif " (voir plus bas). On oppose à ces différents types le dispositif direct, qui ne réalise aucun effet de focalisation.

8 C'est une caractéristique commune à tous les éléments régis réalisés sous une forme lexicale que de pouvoir être instanciés sous une gamme de plusieurs dispositifs ${ }^{7}$ : pour revenir à notre exemple, on relève qu'il peut être donné sous dispositif direct, la séquence causale se trouvant réalisée en fin de construction sans aucun phénomène d'extraction :

(6) ils ne font pas une faute à chaque mot parce qu'ils sont dans les poids lourds 
et sous une forme particulière que nous désignons « Si-dispositif $»^{8}$ qui est très largement compatible avec les séquences régies introduites par parce que, pour + V. inf, à cause de, grâce à, et quelques autres formes; dans le cas de notre exemple, cela donnerait :

(7) s'ils font une faute à chaque mot ce n'est pas parce qu'ils sont dans les poids lourds

c- La modification par un adverbe paradigmatisant : les éléments qui sont sous la dépendance d'un verbe acceptent régulièrement d'être modifiés par un adverbe paradigmatisant comme surtout, seulement, uniquement, éléments qui "présuppose[nt] l'existence d'un paradigme établi à partir de son noyau » (Nølke, 1993, p.31) ; on pourrait avoir :

(8) c'est surtout/ seulement/ uniquement parce qu'ils sont dans les poids lourds qu'ils font une faute à chaque mot

De même, la négation, présente dans l'exemple initial, pourrait sans difficulté se combiner avec un adverbe comme seulement :

(9) ce n'est pas seulement parce qu'ils sont dans les poids lourds qu'ils font une faute à chaque mot

$11 \mathrm{~d}$-Le développement d'un contraste de modalité : la séquence causale de l'exemple 4 peut se développer sous forme d'une liste contrastive en pas parce que... mais parce que :

(10) c'est pas parce qu'ils sont dans les poids lourds qu'ils font une faute à chaque mot, mais parce qu'ils ne lisent jamais

12 Le contraste peut évidemment être donné avec la modalité assertive en tête (c'est parce que... et pas parce que), comme dans un énoncé du type :

(11) c'est parce qu'ils ne lisent jamais qu'ils font une faute à chaque mot et pas parce qu'ils sont dans les poids lourds

13 Si la construction se réalisait à travers d'autres dispositifs, tels que le dispositif direct ou le Si-dispositif, les possibilités de contraste seraient identiques :

(12) ils ne font pas une faute à chaque mot parce qu'ils sont dans les poids lourds mais parce qu'ils ne lisent jamais

(13) s'ils font une faute à chaque mot ce n'est pas parce qu'ils sont dans les poids lourds mais parce qu'ils ne lisent jamais

\subsection{Configuration macrosyntaxique}

La composante macrosyntaxique définie dans le cadre aixois ${ }^{9}$ envisage les énoncés comme une séquence d'unités ordonnées (Pré-Noyau / Noyau / Post-Noyau) qui s'organisent comme des foyers de modalité. Lorsqu'ils sont réalisés sous une forme canonique, les éléments régis placés sous un dispositif focalisant prennent le statut macrosyntaxique de Noyau ; c'est le cas en (4): la parce que-C clivée constitue le Noyau, c'est-à-dire le constituant qui porte la modalité assertive d'énoncé, et le reste de la construction, typiquement pourvu d'une mélodie plate, a la valeur d'un élément postNoyau :

(14) [c'est pas parce qu'ils sont euh dans les poids lourds $]_{\mathrm{No}}$ [qu'ils font une faute à

chaque mot $]_{\text {Post-No }}$

Le noyau a pour caractéristique de former une unité autonome (Blanche-Benveniste et al., 1990, p.291). E. Cresti (2002, p.17) évoque elle aussi le caractère nécessaire et suffisant de ce type d'élément ${ }^{10}:$ " le comment [...] est nécessaire et suffisant pour accomplir un énoncé ».

16 Dans le cas des constructions clivées, l'un des effets les plus visibles de l'autonomie énonciative du Noyau tient à leur possibilité d'être produites sous une forme raccourcie, 
qui réalise la partie Noyau mais pas la partie Post-noyau (Blanche-Benveniste, 1992) ; c'est ainsi qu'on analyserait la version suivante (entre crochets, la partie post-noyau non réalisée) :

(15) ils font une faute à chaque mot mais c'est pas parce qu'ils sont dans les poids lourds [qu'ils font une faute à chaque mot]

Voici un exemple attesté de forme courte de construction clivée :

(16) moi je préfère une édition originale - mais c'est pas parce qu'elle est originale [oral, CRFP]

à partir duquel il serait possible de restituer la séquence Post-Noyau qui n’a pas été produite :

(17) moi je préfère une édition originale - mais c'est pas parce qu'elle est originale que je la préfere

Il apparaît ainsi que la possibilité pour un élément de pouvoir être placé sous dispositif clivé ne doit pas être considérée comme une propriété « indépendante » mais s'inscrit à l'intérieur d'un faisceau de propriétés d'insertion paradigmatique, qui contribuent à révéler le caractère régi de cet élément ${ }^{11}$.

De manière corollaire, les éléments qui ne sont dotés d'aucune propriété d'insertion paradigmatique ne sont pas non plus compatibles avec le dispositif de clivage. Il en irait ainsi d'un adverbe tel que évidemment dans un énoncé comme :

(18) il a raté son train évidemment

dont le caractère non clivable ( ${ }^{*} c^{\prime}$ est évidemment qu'il a raté son train) est à mettre en relation avec d'autres restrictions telles que son absence de proportionnalité avec une proforme (*il a raté son train ainsi) ou l'impossibilité de développer un contraste (*il a raté son train évidemment et non pas subrepticement).

\subsection{Séquences en parce que non régies et clivabilité}

Comme en attestent les travaux de Debaisieux consacrés à la description de parce que dans le français oral ${ }^{12}$, il apparaît que certains emplois de cette conjonction ne peuvent pas être décrits comme des faits de rection verbale. Cela est le cas par exemple de la séquence parce qu'ils portaient tous des grandes djellabas que j'emprunte à un exemple de Deulofeu (2010, p.191):

(19) c'était des Arabes parce qu'ils portaient tous des grandes djellabas

21 Le fait de porter une djellaba n'est évidemment pas présenté ici comme relié de manière causale au fait d'être arabe, mais est donné comme un indice à partir duquel une inférence peut être construite. Par contraste avec les propriétés qu'on vient de rappeler pour les parce que régis, on note que, dans cet emploi :

La séquence en parce que ne peut pas être pronominalisée au moyen de pourquoi :

(20) *pourquoi est-ce que c'était des Arabes? / *pourquoi était-ce des Arabes?

(parce qu'ils portaient tous des grandes djellabas)

La mise sous dispositif focalisant est exclue, qu'il s'agisse du clivage :

(21) ? c'est parce qu'ils portaient tous des grandes djellabas que c'était des Arabes ou du «Si-dispositif » :

(22) *si c'était des Arabes c'est parce qu'ils portaient tous des grandes djellabas La séquence en parce que ne peut pas être développée sous forme de liste contrastive :

(23) *c'était des Arabes parce qu'ils portaient tous des grandes djellabas, et non pas parce qu'ils étaient bronzés 
une construction « support» :

[c'était des Arabes] [parce qu'ils portaient tous des grandes djellabas]

construction 1 construction 2 (associée à la précédente)

On renonce à voir dans ce type d'emploi non régi un exemple de «subordination »: la relation entre les deux constructions successives est de nature parataxique et non pas hypotaxique.

\section{Caractéristiques syntaxiques des constructions $C P P Q Q^{13}$}

\subsection{La séquence en parce que n'est pas régie}

A présent qu'a été rappelée la distinction bien connue entre deux types de constructions en parce que, l'un comme élément régi compatible avec le dispositif clivé, et l'autre comme élément non régi qui est dépourvu de toute propriété d'insertion paradigmatique et qui se trouve associé à une construction-support, nous revenons aux exemples (1-3) initiaux afin de nous demander sur quels points ils diffèrent des constructions clivées mentionnées jusqu'ici. On remarque à leur propos :

a-Certaines limitations liées au choix du dispositif : la plus manifeste est l'impossibilité, qui nous paraît absolue, de recourir au Si-dispositif ${ }^{14}$ :

(24) *si tout de suite notre langue va disparaître c'est pas parce qu'on emploie quelques mots d'anglais

(25) *si un phénomène perd en nocivité ce n'est pas parce qu'il est banalisé

(26) *si une pratique est sans danger ce n'est pas parce qu'elle est répandue

La recevabilité de la réalisation sous forme " directe » est plus délicate à évaluer faute d'exemples attestés fiables ; les exemples (2-3) pourraient sans doute être réalisés sans les marques de clivage, notamment si on leur adjoignait un adverbe du type nécessairement ou forcément :

(27) un phénomène ne perd pas nécessairement en nocivité parce qu'il est banalisé

(28) une pratique n'est pas forcément sans danger parce qu'elle est répandue

b- La proportionnalité avec une proforme présente également des particularités: en dehors de pour ça, qui paraît aussi bien attesté avec les constructions CPPQQ qu'avec les clivées contrastives ${ }^{15}$, on relève la possibilité de faire usage de pour autant :

(29) c'est vrai qu'on emploie quelques mots d'anglais, mais c'est pas pour autant que tout de suite euh notre langue euh elle va disparaître

(30) un phénomène peut bien être banalisé, ce n'est pas pour autant qu'il perd en nocivité

c- La seconde séquence ne peut absolument pas être développée sous forme de liste contrastive :

(31) *c'est pas parce qu'on emploie quelques mots d'anglais que notre langue va disparaître mais parce que son statut de langue internationale est en train de s'affaiblir

(32) *ce n'est pas parce qu'un phénomène est banalisé qu'il perd en nocivité mais pour une autre raison

d- La réalisation sous forme raccourcie n'est pas possible : 
(33) *notre langue va disparaître mais c'est pas parce qu'on emploie quelques mots d'anglais

(34) *une pratique est sans danger mais ce n'est pas parce qu'elle est répandue

e- La modification par un adverbe paradigmatisant est absente de nos relevés et ne nous semble pas naturelle :

(35) ? c'est pas uniquement parce qu'on emploie quelques mots d'anglais que notre langue va disparaître

(36) ? ce n'est pas seulement parce qu'un phénomène est banalisé qu'il perd en nocivité

f- A côté de ces critères paradigmatiques, nous signalons un fait de nature syntagmatique: les constructions organisées sous dispositif clivé (voir point 1.1) paraissent accepter certaines formes d'enchâssement, telles que celles avec la conjonction $s i$; à partir de (4), on pourrait proposer par exemple :

(37) si [ce n'est pas parce qu'ils sont dans les poids lourds qu'ils font une faute à

chaque mot], je me demande bien pour quelle raison ça pourrait être

Nous reconnaissons que de telles formes, sans doute un peu "forcées ", n'apparaissent pas dans les corpus constitués que nous avons consultés; Internet offre toutefois quelques exemples qui ont la particularité de réaliser le dispositif clivé sous sa forme raccourcie $^{16}$ :

(38) Si [ce n'est pas parce que ta PS3 est posée trop près d'une grosse source de chaleur (qu'elle fait ce bruit)], c'est anormal. [écrit, internet, forum de discussion informatique]

(39) Si [ce n'est pas parce que je suis mauvais (que j'ai cette maladie)], alors pourquoi j'ai cette maladie? [écrit, internet, site médical sur les troubles anxieux]

(40) Si [ce n'est pas parce que je lui plais (qu'il m'invite)], alors pourquoi $\mathrm{m}$ a t-il invité? [écrit, internet, courrier du cœur]

De telles manipulations paraissent totalement irrecevables dans le cas des constructions $\mathrm{CPPQQ}$; à partir de (2), on ne peut guère imaginer de suite valable à une séquence comme :

(41) si [ce n'est pas parce qu'un phénomène est banalisé qu'il perd en nocivité], ... ?

Lorsque si apparaît en position post-verbale ${ }^{17}$ (je me demande si), on relève de la même façon que l'enchâssement est parfaitement acceptable dans le cas du dispositif clivé :

(42) je me demande si [c'est pas parce qu'ils sont dans les poids-lourds qu'ils font une faute à chaque mot]

mais reste impossible à partir de (1-3) ; la version suivante, si elle est envisageable, a pour effet inévitable de faire interpréter la séquence enchâssée comme une construction sous dispositif clivé (avec l'effet de contraste qui lui est associé), et non comme la construction CPPQQ d'origine :

(43) je me demande si [ce n'est pas parce qu'un phénomène est banalisé qu'il perd en nocivité]

Nous ne développerons pas ce point davantage, mais insistons sur le fait que conjointement à certaines caractéristiques d'ordre paradigmatique, les constructions CPPQQ paraissent connaître d'autres restrictions de nature syntagmatique, qu'il conviendra d'examiner plus en détail.

Nous résumons dans le tableau suivant les propriétés des parce que-C réalisées dans les deux configurations qui ont été présentées: a) sous dispositif de clivage, à partir de l'exemple c'est parce qu'il faisait beau que je suis sorti ; b) dans une construction CPPQQ, à partir de l'exemple ce n'est pas parce qu'un phénomène est banalisé qu'il perd en nocivité : 


\begin{tabular}{|c|c|c|}
\hline Propriétés & $\begin{array}{l}\text { Dispositif clivé } \\
\text { c'est parce qu'il faisait } \\
\text { beau que je suis sorti }\end{array}$ & $\begin{array}{l}\text { Construction CPPQQ } \\
\text { ce n'est pas parce qu'un phénomène est banalisé } \\
\text { qu'il perd en nocivité }\end{array}$ \\
\hline $\begin{array}{l}\text { Proportionnalité à } \\
\text { une proforme }\end{array}$ & $\begin{array}{l}\text { c'est pour cette raison } \\
\text { / pour cela que je suis } \\
\text { sorti } \\
\text { pour autant impossible: } \\
\text { *'est pour autant que }^{\text {c'e suis sorti }}\end{array}$ & $\begin{array}{l}\text { pour cette raison semble exclu } \\
\text { pour autant possible : ce n'est pas pour autant } \\
\text { qu'il perd en nocivité }\end{array}$ \\
\hline $\begin{array}{l}\text { Passage au dispositif } \\
\text { direct }\end{array}$ & $\begin{array}{l}\text { je suis sorti parce qu'il } \\
\text { faisait beau }\end{array}$ & $\begin{array}{l}\text { peut-être possible, surtout avec l'ajout } \\
\text { d'adverbes comme forcément, nécessairement }\end{array}$ \\
\hline $\begin{array}{lll}\text { Passage } & \text { au } & S i- \\
\text { dispositif } & & \end{array}$ & $\begin{array}{l}\text { si je suis sorti c'est } \\
\text { parce qu'il faisait beau }\end{array}$ & $\begin{array}{l}\text { impossible : *si un phénomène perd en } \\
\text { nocivité ce n'est pas parce qu'il est banalisé }\end{array}$ \\
\hline $\begin{array}{l}\text { Modification par un } \\
\text { adverbe } \\
\text { paradigmatisant }\end{array}$ & $\begin{array}{l}\text { c'est uniquement parce } \\
\text { qu'il faisait beau que je } \\
\text { suis sorti }\end{array}$ & $\begin{array}{l}\text { difficile :? ce n'est pas uniquement parce } \\
\text { qu'un phénomène est banalisé qu'il perd en } \\
\text { nocivité }\end{array}$ \\
\hline $\begin{array}{l}\text { Développement d'un } \\
\text { contraste de modalité }\end{array}$ & $\begin{array}{l}\text { c'est parce qu'il faisait } \\
\text { beau que je suis sorti, } \\
\text { et non pas parce qu'on } \\
\text { me l'a demandé } \\
\text { ce n'est pas parce qu'il } \\
\text { faisait beau que je suis } \\
\text { sorti, mais parce qu'on } \\
\text { me l'a demandé }\end{array}$ & $\begin{array}{l}\text { impossible :*ce n'est pas parce qu'un } \\
\text { phénomène est banalisé qu'il perd en nocivité } \\
\text { mais pour une autre raison }\end{array}$ \\
\hline $\begin{array}{l}\text { Réalisation du clivage } \\
\text { limitée à la séquence } \\
\text { Noyau }\end{array}$ & $\begin{array}{l}\text { je suis sorti mais [c'est } \\
\text { parce qu'il faisait beau] } \\
\text { No }\end{array}$ & $\begin{array}{l}\text { impossible : *un phénomène perd en nocivité } \\
\text { mais [ce n'est pas parce qu'il est banalisé }]_{\mathrm{No}}\end{array}$ \\
\hline Enchâssement avec si & $\begin{array}{l}\text { je me demande si c'est } \\
\text { parce qu'il faisait beau } \\
\text { que tu es sorti }\end{array}$ & $\begin{array}{l}\text { Impossible sans changer de structure : *je me } \\
\text { demande si ce n'est pas parce qu'un } \\
\text { phénomène est banalisé qu'il perd en nocivité }\end{array}$ \\
\hline
\end{tabular}

On conçoit aisément la raison pour laquelle la plupart des manipulations présentées dans la colonne de droite du tableau ci-dessus livrent des versions irrecevables: elles ont comme effet de présenter le contenu de la construction introduite par que comme un fait présupposé, qui devrait être accepté comme vrai sans discussion : dans les versions si un phénomène perd en nocivité ce n'est pas parce qu'il est banalisé, ce n'est pas uniquement parce qu'un phénomène est banalisé qu'il perd en nocivité, ou ce n'est pas parce qu'un phénomène est banalisé qu'il perd en nocivité mais pour une autre raison, le fait qu'un phénomène perde en nocivité semble tenu pour acquis. Or, dans l'énoncé d'origine (ex. 2) il est manifeste, au contraire, que la perte en nocivité dont il est question est présentée comme fortement discutable. Nous reviendrons sur l'interprétation que doivent recevoir ces constructions; 
pour l'instant nous remarquerons que, sur le plan grammatical, les énoncés (1-3) dans lesquels l'élément clivé n'est doté d'aucune propriété d'insertion paradigmatique et paraît également connaître des restrictions liées à l'enchâssabilité ne peuvent pas être assimilés aux formes de «dispositif clivé » décrites par le GARS. En d'autres termes, les séquences en parce que présentes dans ces structures ne peuvent pas être analysées comme des éléments régis par le verbe mais s'apparentent à des constituants non régis ${ }^{18}$.

Une telle analyse se vérifie assez clairement si l'on part de séquences en parce que manifestement non régies (celles, par exemple, qui déclenchent une interprétation inférentielle et non causale, et qui réagiraient négativement aux tests donnés plus haut) ; on vérifie que, quoique non régies, elles semblent accepter d'entrer dans notre structure; à partir de :

(44) il a plu parce que le trottoir est mouillé

(45) il est à la fac parce que sa voiture est dans le parking

(46) c'était des Arabes parce qu'ils portaient tous des grandes djellabas il parait possible de proposer :

(47) ce n'est pas parce que le trottoir est mouillé qu'il a (forcément) plu

(48) ce n'est pas parce que sa voiture est dans le parking qu'il est (pour autant) à la fac

(49) ce n'est pas parce qu'ils portaient tous des grandes djellabas que c'était (nécessairement) des Arabes

La recevabilité de ces exemples illustre une différence essentielle entre le dispositif de clivage et la structure en ce n'est pas parce que... que étudiée dans cet article : alors que le premier n'est susceptible d'affecter que les éléments qui sont sous la dépendance syntaxique d'un verbe (tels que les parce que régis), la seconde semble parfaitement compatible avec les constructions en parce que qui sont dépourvues de tout lien de rection syntaxique avec un verbe.

\subsection{La séquence en parce que n'est pas un simple "élément associé "}

Il convient cependant de s'interroger plus précisément sur le statut non régi de ces séquences en parce que; en effet, les occurrences de parce que non régis telles qu'elles ont été décrites pour le français oral (voir notamment les travaux de Debaisieux) présentent certaines caractéristiques qui les distinguent fortement des structures étudiées ici ; les points suivants peuvent être signalés :

a- la relation entre la séquence en "parce que » et sa construction-support : Debaisieux (2004) observe que la séquence en parce que non régie est parfois « physiquement séparée de sa base $»^{19}$ et constitue une énonciation indépendante, comme dans la séquence parce que une salade ça se garde pas vraiment qui forme un tour de parole autonome :

(50)

L1 donc pour les salades vous êtes obligés d'être livrés tous les jours alors

L2 enfin non pas vraiment tous les jours mais euh -

L1 parce que une salade ça se garde pas vraiment (MacDo, Debaisieux 2004)

Cela n'est évidemment pas possible avec nos structures, qui restent très étroitement intégrées à la construction qui suit au moyen des marqueurs c'est... que et qui de ce fait sont strictement non-autonomisables.

A noter que dans notre structure, la construction à laquelle est associée la construction en parce que est toujours une construction verbale, ce qui n'est pas toujours le cas avec les non régies typiques : 
(51) ce soir sandwiches au jambon pour tout le monde parce que je n'ai pas eu une minute pour m'arrêter au supermarché [ex. inventé] mais

(52) *c'est pas parce que je n'ai pas eu une minute pour m'arrêter au supermarché que ce soir sandwiches au jambon pour tout le monde

b- La forme de la construction introduite par "parce que»: comme on sait (Lakoff 1984, Deulofeu 2010, Debaisieux 1995, 2004, 2007) une séquence en parce que non régie peut introduire un énoncé "racine» doté de sa propre modalité d'énoncé, prenant par exemple la valeur d'une question rhétorique (ex.53), d'une impérative (ex.54) ou d'une exclamative (ex.55) :

(53) on est influençable par rapport à l'anglais finalement parce que pourquoi emprunter des mots euh à l'anglais et pas à l'espagnol ou à l'allemand

[oral, corpaix]

(54) avec tout le quartier on était descendu + pour euh dire qu'on ne voulait pas de la piste cyclable + ben non + parce que regardez tout ce qui s'est produit [oral, CRFP] (55) j'ai travaillé vingt-cinq ans dans les fleurs voyez c'est pas une journée hein alors les fleurs elles me connaissent et moi aussi et les roses hein parce que qu'estce que je me suis piqué les mains avec ces bon sang de roses hein [oral, corpaix]

Comme le souligne justement Deulofeu (2010, p. 193) : «Les unités de type conjonction ou préposition, lorsqu'elles ne sont pas elles-mêmes régies, peuvent introduire des organisations macro complexes en violation des règles de sous-catégorisation ». Or les constructions CPPQQ, pour non régies qu'elles soient, ne peuvent pas pour autant violer les règles de sous-catégorisation; par exemple elles ne peuvent pas porter de modalité d'énoncé :

(56) *c'est pas parce que pourquoi emprunter des mots à l'anglais qu'on est influençable par rapport à l'anglais

(57) *c'est pas parce regardez tout ce qui s'est produit qu'on ne voulait pas de la piste cyclable

Les exemples tirés de corpus oraux révèlent en outre que les séquences causales non régies en relation d'association avec une construction-support prennent fréquemment une forme macrosyntaxique à topique, comme l'exemple suivant, dans lequel la conjonction est suivie par une séquence de plusieurs éléments détachés notés en italiques:

(58) je suis pas tout à fait d'accord parce que moi je vois dans les dans les cours il y a des profs c'est vrai tu as raison ils sont c'est très fluides ça + ça chante c'est très joli [corpaix]

Or dans nos structures, la construction introduite par parce que prend la forme d'une construction à verbe tensé canonique, dans laquelle la conjonction est le plus souvent suivie de la séquence [Sujet-Verbe] ; un exemple typique pourrait être :

(59) c'est pas parce que je fais des études de Sciences du Langage que je dois forcément bien parler [oral, corpaix]

Il est évident que les différences constatées entre les parce que non régis décrits par Debaisieux et ceux apparaissant dans les structures CPPQQ sont liées au fait que dans le premier cas, les parce que-C apparaissent à droite de leur construction de rattachement, dans une relation de pure parataxe, alors que dans le second cas, elles leur sont antéposées et ont une forme de rattachement marquée par des morphèmes (c'est... que ${ }^{20}$. 
a nature de la conjonction : les séquences en parce que non régies ont habituellement de bonnes équivalences avec des conjonctions spécialisées dans l'introduction de syntagmes non régis, telles que puisque, vu que, étant donnéque :

(60) il est à la fac parce que / puisque / vu que / étant donné que sa voiture est dans le parking

Ces conjonctions sont totalement exclues de notre structure ${ }^{21}$ :

(61) c'est pas parce qu'on n'a rien à dire qu'il faut fermer sa gueule [titre de film] mais

(62) *c'est pas puisque on n'a rien à dire qu'il faut fermer sa gueule

(63) *c'est pas vu qu'on n'a rien à dire qu'il faut fermer sa gueule

En résumé, les séquences non régies en parce que qui ont été le plus souvent décrites pour le français sont situées à droite de leur base, qui n'est pas forcément verbale; elles peuvent constituer une énonciation autonome, avec éventuellement un changement de modalité d'énoncé par rapport à leur construction-support; elles peuvent avoir des caractéristiques de «main clause »; et en tout état de cause elles ne sont pas obligatoires. Comme le résume Debaisieux (2004), « aucune contrainte formelle ne semble peser sur la construction base, ni sur la construction en parce que ». Dans les constructions CPPQQ, les parce que-C connaissent au contraire un certain nombre de contraintes : elles ont une place linéaire fixe et morphologiquement marquée, n'ont pas d'autonomie énonciative par rapport à la seconde construction, prennent nécessairement la forme d'une construction verbale tensée sans présenter de phénomène de "main clause ", n'ont pas d'équivalences avec d'autres conjonctions (telles que puisque ou vu que) qui introduisent fréquemment les séquences non régies.

Par conséquent, les faits dégagés dans le paragraphe précédent semblent interdire de réduire ces séquences en parce que à ce que les chercheurs du GARS ont désigné sous le terme d'associés à une construction, tout comme les points étudiés en 2.1 nous empêchent de considérer qu'elles réaliseraient un élément de la rection d'un verbe sous une forme clivée; nous devons conclure que la distinction entre rection et association (ou en d'autres termes, subordination et parataxe) n'offre pas un cadre adéquat pour rendre compte des propriétés observées. Nous proposerons d'analyser les constructions CPPQQ comme une structure spécifique, dans laquelle la séquence non régie en parce que s'unit avec la seconde construction au sein d'une même unité macrosyntaxique.

\subsection{Pour une analyse par « regroupement macrosyntaxique »}

Nous proposerons d'analyser les structures à l'étude comme une forme de regroupement macrosyntaxique entre une construction $\mathrm{A}$ et une construction $\mathrm{B}$, les formes c'est et que marquant le regroupement entre les deux séquences. Le morphème négatif est donné entre parenthèses dans la schématisation ci-dessous, car comme on le verra plus loin (et contrairement à ce qu'on pourrait penser au vu des exemples donnés jusqu'ici), la réalisation de la structure n'implique pas nécessairement la présence d'une négation:

[ c'est (pas) [parce qu'on n'a rien à dire $]_{\text {Constr.A }} q$ ' [il faut fermer sa gueule $\left.]_{\text {Constr. } B}\right]$

Noyau

a- La structure est absolument binaire : les deux séquences doivent être réalisées, ce qui est différent du dispositif clivé dont on a rappelé que la séquence de droite, qui réalise le post-Noyau macrosyntaxique, peut très bien ne pas être produite (cf. ex. 16). 

d'intégration intonative et de symétrie quant à la syntaxe interne des deux constructions successives invitent à analyser le regroupement de constructions comme un noyau macrosyntaxique unique. A certains égards, il serait tentant de voir dans ces structures un type particulier d'organisation corrélative: comme dans les corrélatives typiques (telles que celles en plus... plus), on a bien affaire, selon l'analyse que nous proposons, à une suite de deux constructions verbales n'entretenant aucune relation de rection syntaxique, mais qui se trouvent "attelées » l'une à l'autre grâce à des marqueurs spécifiques $^{23}$.

\subsection{Portée de la négation et interprétation pragmatique} présence de l'élément négatif (ne) pas, qui encadre le verbe être dans la plupart des exemples recensés. Cet élément pose un problème spécifique d'interprétation. Afin d'en dire deux mots, nous allons à nouveau partir du cas du dispositif clivé à effet de contraste (voir énoncé 4) pour revenir par la suite aux structures CPPQQ.

On sait que les énoncés sous dispositif direct, qui s'organisent en un seul noyau macrosyntaxique, présentent des ambiguïtés potentielles vis-à-vis de l'interprétation de la portée de la modalité ; à partir de ${ }^{24}$ :

(67) je ne suis pas allé à Paris parce qu'on me l'a demandé

(68) ils n'ont pas déposé de motion de censure pour faire plaisir au gouvernement 
68 En ce cas, on comprend que l'individu dont il est question en (67) n'est pas allé à Paris, mais ailleurs qu'à Paris, ou nulle part. Et cela implique que la séquence causale introduite par parce que n'est pas atteinte par le sémantisme de la négation. En (68), on devra comprendre qu'aucune motion de censure n'a été déposée.

Interprétation $b:$ la négation porte sur le rapport entre le verbe et l'ajout à valeur causale ( parce qu'on me l'a demandé en (67), pour faire plaisir au gouvernement en (68)).

Ici c'est l'inverse : il est dit en (67) que la personne en question est bien allée à Paris, non pas parce qu'on le lui a demandé, mais pour une autre raison; et en (68), que si une motion de censure a bien été déposée, ce n'est pas dans le but de complaire au gouvernement mais pour un autre motif.

71 Ces difficultés liées au calcul de la portée de la négation disparaissent avec le dispositif clivé (comme avec les autres dispositifs de la rection), dont la caractéristique est précisément de segmenter la construction en deux domaines macrosyntaxiques (dans ce cas, [noyau + post-Noyau]) morphologiquement repérables grâce aux marqueurs c'est et $q u-$, et qui indiquent à l'intérieur de quel constituant macro doit être calculée la portée de la négation :

Dans l'interprétation a-, on aurait :

(69) c'est parce qu'on me l'a demandé que je ne suis pas allé à Paris

(70) c'est pour faire plaisir au gouvernement qu'ils n'ont pas déposé de motion de censure

Et dans l'interprétation b- :

(71) ce n'est pas parce qu'on me l'a demandé que je suis allé à Paris

(72) ce n'est pas pour faire plaisir au gouvernement qu'ils ont déposé une motion de censure

74 Ainsi, dans les énoncés sous dispositif clivé, la présence d'une négation initiale, placée en tête de l'élément Noyau (c'est pas... qu-) indique que c'est dans ce Noyau que doit être recherché l'élément nié.

[c'est pas parce qu'on me l'a demandé] [que je suis allé à Paris]

Noyau : domaine d'interprétation de la portée négative PostNoyau

75 En revanche, dans le cas des structures CPPQQ, il apparaît que la forme pas n'a pas une portée restreinte à la seule séquence en parce que, mais "couvre » l'ensemble de la structure : ce qui est nié, c'est la relation entre la construction $\mathrm{A}$ et la construction $\mathrm{B}$, à savoir, l'idée que le procès décrit dans la seconde séquence puisse être inféré à partir de l'état de fait donné dans la première. Une glose approximative pourrait en être : «il est incorrect d'en venir à la conclusion il faut fermer sa gueule à partir de la prémisse on n'a rien à dire. " (cf. Kanetani, 2004). C'est ainsi que, pragmatiquement, les deux constructions présentes dans le regroupement peuvent s'analyser comme une séquence [prémisse conclusion] (Hirose, 1991):

c'est pas [parce qu'on n'a rien à dire] qu'[il faut fermer sa gueule]

Constr. A (prémisse) Constr. B (conclusion)

Ces constructions paraissent donc être fondamentalement liées à un acte de réfutation d'une inférence de la part de l'énonciateur, et constituent de ce fait des structures dialogiques par essence, ce que confirme le fait qu'elles se réalisent comme des énoncés racine et qu'elles connaissent de fortes restrictions quant à leurs possibilités d'enchâssement (cf. 2.1. point f.) ${ }^{25}$ 
77 Dans les exemples tirés de corpus, le rejet de l'inférence est souvent lié à l'emploi d'adverbes qui expriment l'idée que le lien entre prémisse et conclusion n'est pas « forcé », pas « nécessaire », pas « fatal » :

(73) ce n'est pas parce que l'on remonte loin dans le temps que l'on va nécessairement du complexe au simple. [écrit, CERF]

(74) Ce n'est pas parce que l'on a tort qu'il faut nécessairement payer [Frantext: Boulanger D., 1962, 147]

(75) c'est pas parce que je fais des études de Sciences du Langage que je dois forcément bien parler [oral, corpaix]

(76) Ce n'est pas parce que c'est du brut que c'est forcément du mauvais [Frantext:

Billetdoux M., 2006, 33]

De tels adverbes font d'ailleurs partie des "ingrédients " propres à cette construction. Avec des structures manifestement interprétables comme des clivées traditionnelles, ils ne passent pas :

(77) ? hier, c'est parce qu'il a plu que j'ai forcément pris mon parapluie

On relève dans les corpus que la réfutation exprimée par ces constructions est souvent liée à certains domaines sémantiques particuliers, tels que :

80 - la relation entre un fait et les obligations qu'il pourrait impliquer (cf. des verbes comme devoir, être obligé, falloir dans ces exemples) :

(78) c'est pas parce que je fais des études de Sciences du Langage que je dois forcément bien parler [oral, corpaix]

(79) c'est pas parce que euh on veut faire euh une unité économique qu'on est obligé euh de parler tous la même langue et de faire disparaître toutes les langues qui existent [oral, corpaix]

(80) tout d'abord, ce n'est pas parce que dans les secteurs économiques où règne le libéralisme on perpétue une tradition de surexploitation du travailleur ordinaire qu'il faudrait étendre le vent de la flexibilité à l'ensemble des professions. Le contraire serait plutôt souhaitable. [écrit, CERF]

81 - la relation entre un fait et les droits qu'il implique :

(81) Expliquons à nos enfants que ce n'est pas parce qu'on est « sain » qu'on peut se dispenser du préservatif. [écrit, net]

(82) Ce n'est pas parce que nous manquons encore d'essence et de pneumatiques que nous avons le droit, pour autant, de négliger l'avenir de la route et de l'automobile. [Frantext : Pineau C., 1950, 125]

- la relation entre un fait actuel et ce qu'il implique pour l'avenir :

(83) ce n'est pas parce que le Soleil s'est levé qu'il se lèvera demain [écrit, CERF]

(84) c'est pas parce qu'on entend beaucoup de chansons anglaises que on va se mettre à plus parler français [oral, corpaix]

83 - la relation entre un fait et son caractère moralement acceptable :

(85) c'est pas parce que je dis et que j'annonce tout ceci que je prévois tout cela que je juge que c'est bien [oral, corpaix]

(86) Mais ce n'est pas parce qu'une loi est adoptée qu'elle est juste ! [écrit, CERF]

(87) Et ce n'est pas parce que Barre, Léotard ou Delors (les centristes en somme) votent non qu'ils ont raison. Ils se sont toujours trompés sur tout. [écrit, CERF]

\subsection{Au-delà de la négation}

84 Il est intéressant de constater que si ces structures permettent à l'énonciateur de réfuter la validité d'une inférence, elles ne lui permettent pas en revanche d'exprimer qu'il y adhère : un énoncé comme 
(88) c'est parce qu'on n'a rien à dire qu'il faut fermer sa gueule ne peut sans doute être interprété que comme une forme de clivage contrastif, dans laquelle la parce que-C est régie par le verbe de la seconde séquence, et qu'on pourrait mettre en rapport avec :

(89) s'il faut fermer sa gueule c'est parce qu'on n'a rien à dire ou que l'on pourrait accompagner d'un élément de contraste :

(90) c'est parce qu'on n'a rien à dire qu'il faut fermer sa gueule ; pas pour une autre raison

Mais cela ne signifie pas pour autant que la forme négative est grammaticalement requise. Considérons les exemples inventés suivants :

(91) Alors comme ça, tu penses vraiment quec'est parce qu'on n'a rien à dire qu'il faudrait fermer sa gueule !

(92) S'il s'imagine que c'est parce qu'on n'a rien à dire qu'il faut fermer sa gueule !

(93) Depuis quand ce seraitparce qu'on n'a rien à dire qu'il faudrait fermer sa gueule?

Ce qui permet à ces énoncés de bien fonctionner malgré l'absence de la négation, c'est qu'ils paraissent s'inscrire dans un contexte polémique qui rend manifeste la désapprobation ou l'incrédulité de l'énonciateur par rapport à l'inférence donnée dans la construction $\mathrm{CPPQQ}^{26}$.

En revanche, à l'inverse de verbes subjectifs comme " penser » ou « s'imaginer », un verbe comme savoir, qui présente l'état de fait évoqué comme non soumis à discussion, paraît mauvais ; comparer :

(94) il s'imagine que c'est parce qu'on n'en parle pas que ça n'existe pas

(95) ? il sait bien que c'est parce qu'on n'en parle pas que ça n'existe pas

Pour la même raison, les formes interro-négatives, par lesquelles l'énonciateur demande à l'auditeur d'entériner son propre point de vue, nous paraissent également assez bizarres:

(96) ? est-ce que tu ne penses pas que c'est parce qu'on n'en parle pas que ça n'existe pas?

\section{Conclusion}

Cet article a permis d'illustrer par de nouveaux exemples un fait déjà bien connu : les formes clivées ne sont pas toujours porteuses d'un effet de sens contrastif ${ }^{27}$. Comme le soulignait Blanche-Benveniste (2006),

«La construction en c'est...que/qui,... [...] a été analysée comme un 'gallicisme' qui favoriserait la mise en relief, le contraste ou, de façon plus générale, la 'focalisation d'un élément' ou sa 'saillance communicative'. Pendant longtemps, les exemples cités illustraient surtout l'effet de contraste, considéré comme le plus révélateur (en particulier dans les analyses générativistes des années 1990) :

(1) C'est toi qui l'as cassé, ce n'est pas moi.

Certains linguistes, soucieux des exemples réellement attestés, se sont avisés qu'on ne pouvait pas ramener toutes les occurrences de cette construction à des effets de focalisation aussi simples et que la notion d'expressivité ne pouvait pas tout expliquer. »

Doetjes, Rebuschi et Rialland (2003) font une remarque similaire :

«[...] we will take into account a much wider array of data, and show that the typical clefts in which the focus corresponds to the XP and the rest of the sentence is given are but one possible type of cleft sentence [...]» 
91 Le problème reste de savoir si le caractère contrastif ou non contrastif de certaines constructions clivées peut être ramené à une simple question d'organisation informationnelle, liée à la place du focus dans l'énoncé, ou si la structure syntaxique est différente aussi ; dans le cas des énoncés en ce n'est pas parce que... que étudiés dans cet article, nous avons fait l'hypothèse qu'il fallait poser une organisation grammaticale spécifique : les clivées contrastives le plus souvent décrites relèvent des dispositifs de la rection et prennent place parmi une palette de dispositifs qui illustrent tous certaines propriétés d'insertion paradigmatique typiques des éléments régis par un verbe. En revanche, les structures $\mathrm{CPPQQ}$, si nous acceptons d'y voir des formes de clivage, échappent totalement à l'analyse en "dispositif de la rection » : elles ne manifestent pas l'extraction d'un des éléments de la rection verbale mais se laissent plus aisément décrire comme un type particulier de regroupement macrosyntaxique entre deux constructions sans relation de dépendance microsyntaxique ${ }^{28}$. Ce regroupement n'est cependant pas de pure parataxe en raison de la présence des marqueurs de clivage qui concourent à stabiliser la relation entre les deux séquences successives de manière originale. Spécifiques dans leur forme grammaticale, les constructions CPPQQ le sont aussi dans leurs effets pragmatico-sémantiques, qui sont directement liés à l'expression de la réfutation d'une inférence causale.

\section{BIBLIOGRAPHIE}

ABEILLÉ, A., GODARD, D. \& SABIO, F. (2008), « Deux constructions à SN antéposé en français », Actes du Congrès Mondial de Linguistique Française (CMLF'08). Durand J., Habert B. et Laks B. (éds.), p. 2361-2376.

BENDER, E. M. \& KATHOL, A. (2001), « A constructional analysis of just because... doesn't mean .... », Talk presented at the First International Conference on Construction Grammar, April 6-8, 2001, UC Berkeley.

BLANCHE-BENVENISTE, C. (1992), «A propos des énoncés sans verbe : les énoncés réponses », Recherches sur le français parlé, $\mathrm{n}^{\circ} 11$, p. 57-85.

BLANCHE-BENVENISTE, C. (2002), « Macro-syntaxe et micro-syntaxe : les dispositifs de la rection verbale ", in Andersen H.L. \& Nølke H. (éds.), Macro-syntaxe et macro-sémantique, Berne, Peter Lang, p. 95-115.

BLANCHE-BENVENISTE, C. (2006), « Les clivées françaises de type : C'est comme ça que, C'est pour ça que, C'est là que tout a commencé ", Moderna språk, n¹00, p. 273-287.

BLANCHE-BENVENISTE, C. (2010), Le français : usages de la langue parlée, coll. Langues du monde, Leuven/Louvain, Peeters.

BLANCHE-BENVENISTE, C., BILGER M., ROUGET C., VAN DEN EYNDE K. \& MERTENS P. (1990), Le français parlé, études grammaticales, Paris, Éditions du CNRS.

BLANCHE-BENVENISTE, C., DEULOFEU, J., STEFANINI, J. \& VAN DEN EYNDE, K. (1984), L'Approche pronominale et son application au français, Paris, SELAF. 
CRESTI, E. (2002), « Illocution et modalité dans le comment et dans le topic », Preprint $n^{\circ} 2$, LABLITA, U. de Firenze.

DEBAISIEUX, J.-M. (1995), « Le fonctionnement de parce que en français parlé contemporain », in Vetters, C., Actes de la Première Rencontre des jeunes linguistes, p. 33-44.

DEBAISIEUX, J. M. (2004), « Les conjonctions de subordination : mots de grammaire ou mots du discours ? Le cas de parce que ", Revue de Sémantique et de Pragmatique, n¹5/16, p.101-119.

DEBAisieUX, J.-M. (2007), « La distinction entre dépendance grammaticale et dépendance macrosyntaxique comme moyen de résoudre les paradoxes de la subordination ", Faits de Langues, $\mathrm{n}^{\circ} 28$, p. 119-132.

DEULOFEU, J. (1991), « La notion de dépendance syntaxique dans l'approche pronominale », L'information grammaticale, $\mathrm{n}^{\circ} 50, \mathrm{p} .19-24$.

DEULOFEU, J. (2003), «L'approche macrosyntaxique en syntaxe : un nouveau modèle de rasoir d'Occam contre les notions inutiles?», Scolia, n²16,p. 77-95.

DEULOFEU, J. (2010), « La greffe d'un énoncé sur une construction : une combinaison originale de parataxe et de rection », in Béguelin, M.-J., Avanzi, M. \& Corminboeuf, G. (éds.), La parataxe, Berne, Peter Lang, p. 175-208.

DoETJES, J., REBusChI, G., RIALland, A. (2003), « Cleft sentences, Chapter 4 » in Corblin, F. \& Hulk, A. (éds.), A Semantic Description of French, Stanford, CSLI Publications, p. 529-552.

HIROSE, Y. (1991), « On certain nominal use of Because-Clauses: Just because Because-Clauses can substitute for That-Clauses does not mean that this is always possible, » English Linguistics, $\mathrm{n}^{\circ} 8, \mathrm{p}$. 16-33.

KANETANI, M., 2004, « A note on because-clauses », in Kobukata Y. \& Tamura T. (éds.), TES, n²3, p. 91-98.

LAKOFF, G. (1984), « Performative Subordinate Clauses » Berkeley Linguistics Society, n¹0, p. 472-480.

LAMBRECHT, K. (1988), « Presentational cleft-constructions in Spoken French », in Haiman, J. \& Thompson, S.A., Clause combining in grammar and Discourse, Amsterdam, Benjamins, p. 135-179. LAMBRECHT, K. (2001), « A Framework for the analysis of Cleft construction », Linguistics, n³9-3, p. 463-516.

NøLKE, H. (1993), Le regard du locuteur. Pour une linguistique des traces énonciatives, Paris, Kimé. SABIO, F. (1996), Description prosodique et syntaxique du discours en français. Données et hypothèses, thèse de Doctorat, Université de Provence.

SABIO, F. (à par.), « Description de quelques configurations en Si dans le français contemporain », in Debaisieux J.-M. (dir.), Apports des analyses sur corpus : subordination et pseudo-subordination en français contemporain, Paris, Hermès.

TESTON, S. (2006), Propriétés topologiques et distributionnelles des constituants non régis. Application à une description syntaxique des particules discursives, Thèse de Doctorat, Université de Provence. 


\section{NOTES}

1. Les exemples attestés proviennent de corpus oraux (base " corpaix », Corpus de Référence du Français Parlé [CRFP]) ; et écrits (corpus CERF, élaboré par Véronis). Quelques exemples sont tirés de Frantext et du Net. Les énoncés oraux sont fournis sans majuscule ni ponctuation.

2. Les analyses menées à partir des années 1980 ont très largement privilégié l'étude de ces clivages contrastifs, liés à la focalisation d'un élément, que ce soit d'un point de vue syntaxique, pragmatique ou prosodique: voir par ex. Nølke (1993); Lambrecht $(1988,2001)$; Doetjes, Rebuschi \& Rialland (2003).

3. Cf. Blanche-Benveniste et al. (1984); Blanche-Benveniste et al. (1990); Deulofeu (1991).

4. Nous ne mentionnons ici que les critères pouvant être appliqués aux constructions clivées, qui font seules l'objet de cet article.

5. Cf. Blanche-Benveniste (2002).

6. On désigne ainsi les structures en «il n'y a que... que » du type il n'y a qu'ici qu'il n'a pas plu.

7. Par exemple, la position syntaxique instanciée par à Marie dans il veut parler à Marie peut se réaliser à travers les dispositifs suivants : direct ; antéposé (à Marie il veut parler [et à personne d'autre]) ; clivé (c'est à Marie qu'il veut parler) ; restrictif (il n'y a qu'à Marie qu'il veut parler) ; pseudo-clivé (celle à qui il veut parler c'est [à] Marie).

8. Sabio, à paraître.

9. Cf. Deulofeu (2003).

10. Qu'elle nomme « comment ».

11. On note toutefois que certains éléments, quoique régis par le verbe, supportent mal le clivage : il en va ainsi de certaines Que-Constructions qu'on aurait peu de chance de trouver sous la forme:? c'est qu'il vienne que j'aimerais. Ces difficultés sont prévisibles en partie selon la catégorie de l'élément régi, et en partie selon les caractéristiques lexicales du verbe recteur. Nous ne développerons pas cet aspect ici.

12. Cf. par ex. 1995, 2004, 2007.

13. Afin d'éviter toute confusion, nous désignerons les organisations illustrées par les exemples (1-3) par la formule " $\mathrm{CPPQQ}$ », encouragé en cela par certains linguistes anglo-saxons qui ont l'habitude d'utiliser les initiales «JB-DM » pour désigner la construction équivalente de l'anglais : «just because ... doesn't mean... » (cf. Bender \& Kathol, 2001).

14. Une précision importante: dans la série d'exemples qui suit, le signe ${ }^{*}$ indique moins l'agrammaticalité des structures que leur non-correspondance avec les énoncés de départ (ex. 1-3).

15. Cf. cet exemple de dispositif clivé : « c'est pas pour ça que je crie hein c'est pas parce que je suis sourd [...] mais c'est pour me faire entendre + voilà [...] on crie pour pour se faire entendre » [oral, corpaix] ; et ces exemples de constructions CPPQQ : « maintenant bon ben si on (n') a même pas les moyens de mettre un franc dans un préservatif et ben c'est pas pour ça qu'on doit s'en passer » [oral, corpaix] ; « Je n'aurais même pas remarqué la campagne Perrier si des gens sans doute bien intentionnés n'avaient pas fait tout un foin à son sujet sur ce forum. Alors après, bien sûr, j'y ai fait attention, et l'ai même trouvée assez drôle, mais ce n'est pas pour ça que je vais me mettre à acheter du Perrier » [écrit, CERF]

16. Je restitue entre parenthèses une version de la séquence post-Noyau qui n'a pas été réalisée dans l'énoncé attesté.

17. Avec d'autres conjonctions, l'enchâssement des CPPQQ est bien attesté; par ex.: «Expliquons à nos enfants que ce n'est pas parce qu'on est 'sain' qu'on peut se dispenser du préservatif. » [écrit, CERF]; « donc je l'accepte en tant que tel parce que c'est pas parce que la personne est morte - que je n'ai plus de service à lui rendre » [oral, CRFP]; cette différence de 
comportement confirme que les enchâssements sur la gauche sont plus contraignants que ceux qui se développent dans la partie postverbale.

18. Notre analyse, qui vise à faire des constructions $C P P Q Q$ des organisations spécifiques, s'éloigne de celle proposée par Blanche-Benveniste (2010), qui semble considérer que ces structures peuvent être totalement assimilées aux clivées contrastives ordinaires; elle illustre, par exemple, la notion générale de clivage avec des exemples tels que «ce n'est pas parce que les gens ne peuvent pas faire ceci ou cela qu'il faut les oublier » ou «ce n'est pas parce que vous le dites que ça va s'arrêter » sans mentionner le caractère particulier de ces constructions.

19. Ce serait d'ailleurs aussi le cas des Si-constructions régies lorsqu'elles connaissent une réalisation " différée », en épexégèse.

20. Le phénomène de reprise dialogique associé à ces constructions (voir plus loin) joue sans doute aussi : les formes de citation ont toujours une syntaxe quasiment canonique.

21. Ce qui peut être mis en relation avec le fait que puisque ou vu que ne peuvent pas introduire des éléments focalisés.

22. De même, il paraît peu probable qu'on ait affaire à des séquences analysables comme une succession d'un pré-Noyau et d'un Noyau: en effet, Teston (2006) notait qu'il était fréquent qu'entre un élément pré-Noyau et le Noyau subséquent puissent apparaître des éléments phatiques comme ben ; par exemple « un patois + ben c'est une langue euh orale + + propre euh à une région » [corpaix]; «ce qui me plaît aussi ben c'est quand même de bosser avec les instits parce qu'on apprend plein de choses " [corpaix]. On n'a pas rencontré pour l'instant d'énoncés comme : «? c'est pas parce qu'il est américain ben qu'il est riche ».

23. Les marques du regroupement en revanche s'éloignent du modèle corrélatif canonique dans le sens où, à la différence des énoncés en plus... plus, les marques morphologiques qui produisent l'effet de regroupement - c'est et que - n'ont aucun statut d'élément régi au sein de la construction qui les suit. En outre, Deulofeu nous fait remarquer que l'analyse en corrélation serait problématique dans les cas où la construction $\mathrm{A}$ serait réduite à un élément de type SP ou Adverbe ; cf. des ex. comme «si on (n') a pas d'enseignement de la grammaire de l'orthographe du français euh c'est pas au collège qu'on va savoir écrire » [oral, corpaix] ; «c'est pas maintenant que tu vas devenir une sainte » [écrit, Net]

24. Sabio (1996).

25. Nous avions remarqué (Abeillé, Godard \& Sabio, 2008, p. 2368) que les constructions du type 10 ans il vient d'avoir, que le GARS a décrit comme un "dispositif par antéposition ", prennent des caractéristiques dialogiques assez comparables; nous notions à leur propos : «L'impossibilité de l'enchâssement montre que [cette] construction est associée à un type d'énoncé, et donc, à un acte illocutoire. En fait, elle est associée à un énoncé de reprise, qui est un acte dialogique avec plusieurs sous-types (assertion-de-reprise, question-de-relance, etc.); c'est pourquoi nous l'appelons l'extraction dialogique ".

26. Cf. Bender \& Kathol (2001), qui relèvent ce fait pour les constructions en just because... doesn't mean de l'anglais.

27. Par exemple, parmi les clivées non contrastives, il existe un type assez spécifique qui a été étudié par Blanche-Benveniste (2006), dans lequel l'élément extrait contient une proforme comme c'est comme ça que, c'est ainsi que, c'est pour ça que, c'est là que, dans des exemples du type : «C'est ainsi, mesdames et messieurs les jurés, que Leduc [...] n'a point repris la rue de la République "; "Donc nous avons été amenés à rencontrer des entreprises pour avoir des informations sur les métiers qui nous intéressaient et euh c'est comme ça que j'ai euh contacté une entreprise pour effectuer mon stage pratique " [ex. oraux cités par Blanche-Benveniste]. Dans ces exemples, la partie clivée donne un effet de récapitulation des éléments introduits dans le contexte antérieur : récapitulation de la cause (pour ça), des circonstances (ainsi, comme ça) ou de la situation énonciative (là). 
28. Ce serait aussi le cas des structures en c'est comme ça que..., c'est ainsi que..., c'est pour ça que... évoquées dans la note 27. Pour ces divers types de constructions, nous pourrions parler de « dispositif macrosyntaxique », en opposition aux « dispositifs de la rection » décrits par le GARS.

\section{RÉSUMÉS}

Nous proposons dans cet article d'étudier l'organisation grammaticale des énoncés tels que « ce n'est pas parce qu'une pratique est répandue qu'elle est sans danger ».

Ces structures, qui sont manifestement apparentées aux constructions clivées, possèdent des propriétés grammaticales tout à fait spécifiques qui interdisent selon nous de les assimiler aux clivées « contrastives» les plus souvent décrites : comme nous le montrerons, les syntagmes en parce que qui apparaissent dans la première partie de ces constructions ne peuvent ni être décrits comme des subordonnées canoniques, ni être analysés comme des éléments non régis qui seraient en relation de simple association parataxique avec la construction verbale qui les suit. Nous proposerons de voir dans ces structures un type original de regroupement macrosyntaxique dont la forme est directement reliée à certains effets interactionnels de dénégation d'une inférence causale.

This paper gives an grammatical account of French utterances such as "Ce n'est pas parce qu'une pratique est répandue qu'elle est sans danger", which could be translated in English by "Just because a practice is widespread doesn't mean it is safe".

These structures, which are obviously related to cleft constructions, possess highly specific grammatical properties, and thus they cannot simply be assimilated to the most usually described "contrastive" clefts sentences: as will be shown, the Because-clauses appearing in the first sequence of the constructions cannot either be described as canonical subordinate phrases, or be analyzed as ungoverned elements that share a simple paratactic relationship with the verbal construction following them. We shall suggest that the "C'est pas parce que"- utterances are organized as a particular type of "macrosyntactic" grouping, which is directly connected with certain interactional effects of inference denial.

\section{AUTEUR}

\section{FRÉDÉRIC SABIO}

Aix-Marseille Université, CNRS Laboratoire Parole et Langage UMR 6057

frederic.sabio@orange.fr 